\title{
Top-Down Fabrication of High Quality Gallium Indium Phosphide Nanopillar/disk Array Structures
}

\author{
Dennis Visser \\ Department of Applied Physics \\ KTH Royal Institute of Technology \\ Kista, Sweden \\ dvisser@kth.se \\ Marcin Swillo \\ Department of Applied Physics \\ KTH Royal Institute of Technology \\ Stockholm, Sweden \\ marcin@kth.se
}

\author{
Rinat Yapparov \\ Department of Applied Physics \\ KTH Royal Institute of Technology \\ Kista, Sweden \\ yapparov@kth.se \\ Yohan Désières \\ University of Grenoble Alpes \\ CEA, LETI, MINATEC \\ Grenoble, France \\ yohan.desieres@cea.fr \\ Srinivasan Anand \\ Department of Applied Physics \\ KTH Royal Institute of Technology \\ Kista, Sweden \\ anand@kth.se
}

\author{
Eleonora De Luca \\ Department of Applied Physics \\ KTH Royal Institute of Technology \\ Stockholm, Sweden \\ eldl@kth.se \\ Saulius Marcinkevičius \\ Department of Applied Physics \\ KTH Royal Institute of Technology \\ Kista, Sweden \\ sm@kth.se
}

In this work, top-down fabrication methods for fabricating high optical quality gallium indium phosphide (GaInP) nanopillar/disk arrays are investigated for optoelectronic applications. Time-resolved photoluminescence (TRPL) measurements are used to characterize the fabricated nanostructures and the results are compared to the properties of a reference GaInP 'slab'. Photoluminescence (PL) spectra and carrier lifetimes are characterized for the fabricated GaInP structures embedded in a highly transparent film. Additionally, using GaInP structures on a gallium arsenide (GaAs) substrate the effect of a sulphur-oleylamine based surface passivation procedure is investigated. This was done for the purpose of improving the PL intensities, increase carrier lifetimes and prevent photodegradation by passivating the surface states.

\section{A. Introduction}

III-V semiconductor materials show interesting properties for optoelectronic applications, e.g., high refractive index, direct bandgap, absorption properties and high carrier mobility. For example, GaInP and AlGaInP have been reported for a wide range of applications such as transistors [1], diodes [2], lasers [3], light emitting diodes (LEDs) [4], solar cells [5] and window layers in solar cells [6]. Top-down [7-12] and bottom-up [13-16] fabrication methods for structuring $\mathrm{GaInP}$ have been reported to fabricate nanostructured layers in order to enhance light-matter interactions. Patterning of the initial layer can be beneficial for several features, e.g., absorption enhancement, light extraction enhancement and/or improvement of carrier extraction. However, processing of the layer may lead to a degradation of the optical quality due to surface states and process induced defects. Therefore, it is important to investigate the effect of the fabrication methods on the material properties of GaInP by characterizing the optical/electrical quality of the structures. Important features for these applications are the optical and electrical properties of this patterned material. Bottom-up methods rely on the assembly or growth of a (distorted) starting material, by supplying proper conditions (e.g., reactants, temperature and pressure). Challenges for this method are to control the quality of the material, geometry (size and shape) and correct assembly location. Reported methods are, e.g., gold-seeded growth, self-seeded growth and selective area growth [17]. Top-down methods rely on a starting bulk material, for which the initial quality of the grown (Al)GaInP layer plays an important role. A selective etching process is used on this layer to obtain the desired geometry and spacing of the final structuring. Approaches used for this are: reactive ion etching (RIE), inductively coupled RIE (ICP-RIE) and wet etching [7]. In this work, GaInP nanopillar/disk array structures were fabricated by a combination of a colloidal lithography and an ICP-RIE process in order to obtain nanostructures with a controlled geometry and spacing, and high optical quality. These GaInP nanopillar/disk structures were designed to present low reflection, high absorption as well as high light extraction efficiency in the visible range, with possible applications in order to achieve anti-reflection and absorption enhancement, coloring, sensing, or wavelength down conversion. The fabricated structures were embedded in a highly transparent film in order to study their optical properties, especially their photoluminescence properties. Additionally, a surface passivation procedure was investigated for the structures still on the substrate in order to improve the optical quality of the fabricated structures.

\section{B. Fabrication}

GaInP nanopillar/disk arrays were fabricated from high quality epitaxially grown $\mathrm{Ga}_{0.51} \mathrm{In}_{0.49} \mathrm{P}$ layers, on a $\mathrm{GaAs}$ substrate, having a direct bandgap emission wavelength of $\sim 660 \mathrm{~nm}$; where the structures were obtained from ENT E. A., Poland. As a reference, microdisks were fabricated to mimic a bulk-like GaInP slab. A stack layer of GaInP/GaAs was used in order to obtain substrate-free GaInP structures embedded in a polydimethylsiloxane (PDMS) film. A fabrication process based on a combination of colloidal lithography (CL) or optical lithography and inductively coupled plasma reactive ion etching (ICP-RIE) was used for obtaining the GaInP 

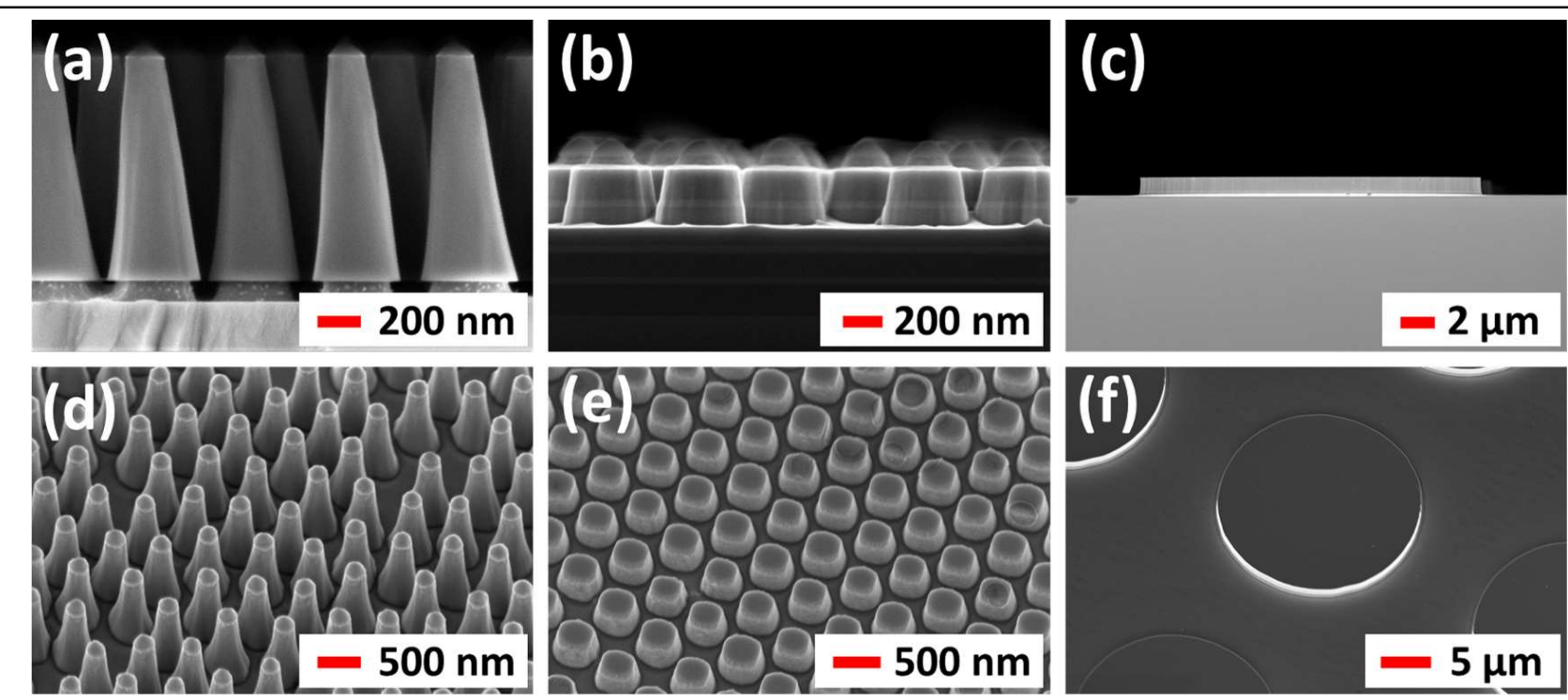

Fig. 1. Scanning electron microscopy (SEM) images of the fabricated GaInP nano- and microstructures. Cross-section images of the ICP-RIE etched (a) nanopillar arrays (with mask and partially wet etched), (b) nanodisk arrays (with mask) and (c) microdisk, are shown. Tilted (30 $\left.{ }^{\circ}\right)$ top view images are shown for the ICP-RIE etched (d) nanopillar arrays, (e) nanodisk arrays and (f) microdisk.

structures; where CL was used for the nanopillar/disk structures and optical lithography for the (reference) microdisk. For the CL, a colloidal solution of silicon dioxide $\left(\mathrm{SiO}_{2}\right)$ nanospheres (Sigma Aldrich; diameter of $\sim 500( \pm 5 \%)$ $\mathrm{nm})$ was used for masking purposes. A thin $\mathrm{SiO}_{2}$ layer (thickness of $\sim 55 \mathrm{~nm}$ ) was first deposited on the initial GaInP layer by plasma-enhanced chemical vapor deposition (PECVD) in order to improve the surface wettability and to serve as an additional hard mask. The $\mathrm{SiO}_{2}$ colloidal solution was then deposited on the surface by a mild spin coating process, resulting in close-packed hexagonal array patches with a homogeneous coverage of several $\mathrm{mm}^{2}$; where the original colloid diameter determines the hexagonal array period. The organized colloidal particles are then size reduced by a RIE process $\left(\mathrm{CHF}_{3}\right.$ flow of $25 \mathrm{sccm}$, RF power of 100 $\mathrm{W}$, pressure of $50 \mathrm{mTorr}$ and an average diameter size reduction rate of $\sim 20 \mathrm{~nm} / \mathrm{min}$ ). For the microdisks, optical lithography patterning was used, where the pattern was transferred from a patterned positive photoresist layer to a 300-nm-thick $\mathrm{SiO}_{2}$ PECVD (masking) layer deposited on the initial GaInP layer. An initial GaInP layer of $1 \mu \mathrm{m}$ thickness was used for the fabrication of the nanopillar arrays and the microdisks, whereas a $200 \mathrm{~nm}$ layer thickness was used for the fabrication of the nanodisk arrays; where the thickness of the layer determines the height of the structures. $\mathrm{A} \mathrm{Cl}_{2} / \mathrm{H}_{2} / \mathrm{CH}_{4}-$ based chemistry was used for the ICP-RIE process in order to etch the micro/nanostructures $\left(\mathrm{Cl}_{2}\right.$ flow of $9 \mathrm{sccm}, \mathrm{H}_{2}$ flow of $5.5 \mathrm{sccm}, \mathrm{CH}_{4}$ flow of $7.5 \mathrm{sccm}$, ICP power of $1 \mathrm{~kW}, \mathrm{RF}$ power of $100 \mathrm{~W}$, temperature of $60^{\circ} \mathrm{C}$, set pressure of 4 mTorr and (calibrated) etch rate of $\sim 170 \mathrm{~nm} / \mathrm{min}$ ). A selectivity between the GaInP: $\mathrm{SiO}_{2}$ mask of $\sim 4-5$ was observed, resulting in a slightly tapered side wall. A thin $(100 \mathrm{~nm}) \mathrm{GaAs}$ sacrificial layer was provided below the GaInP layer. Substrate-free structures are then obtained by using a selective wet etching process to etch away the GaAs layer. For the (partial) sacrificial layer etch, a wet etch chemistry based on $\mathrm{H}_{3} \mathrm{PO}_{4} / \mathrm{H}_{2} \mathrm{O}_{2} / \mathrm{H}_{2} \mathrm{O}$ (ratio of $3: 1: 25$ and etch rate of $\sim 300$ $\mathrm{nm} / \mathrm{min}$ ) is used. To investigate the GaInP structures without the influence of the GaAs substrate, the fabricated substratefree GaInP structures were embedded in a PDMS (Dow Corning, Sylgard 184 Silicone Elastomer) film which was subsequently peeled off from the substrate. The resulting nanopillar arrays have a height of $1 \mu \mathrm{m}$, hexagonal array period of $\sim 500 \mathrm{~nm}$ and a top-bottom diameter of $\sim 150-350$ $\mathrm{nm}$. The nanodisk arrays have a height of $200 \mathrm{~nm}$, hexagonal array period of $\sim 500 \mathrm{~nm}$ and a diameter of $\sim 350 \mathrm{~nm}$. The microdisk has a height of $1 \mu \mathrm{m}$ and diameter of $\sim 20 \mu \mathrm{m}$. Representative scanning electron microscopy (SEM) images of the fabricated structures are shown in Fig. 1. For the sculpting and passivation of the structures, a sulphuroleylamine based treatment was used (1.5\% sulphur solution, temperature of $94{ }^{\circ} \mathrm{C}$ and treatment time of 3.5 hours) [18]. This resulted in a slight removal of the surface material and the formation of chemical bonds between sulphur and GaInP; thereby acting as a passivation layer. These preliminary studies were performed for the ICP-RIE etched GaInP structures still on the substrate.

\section{Optical Characterization}

Lattice matched epitaxially grown GaInP layers have been reported to show good electrical and optical properties such as their minority exciton mobility and diffusion length, absorption dynamics, and PL/Raman properties [19-24]. Characterization of the original $\mathrm{GaInP} / \mathrm{GaAs}$ substrate and the embedded GaInP 'slab' in PDMS by high-resolution X-ray diffraction (HR-XRD) analysis, energy-dispersive X-ray spectroscopy (EDS) and Raman spectroscopy, indicates a proper lattice matched layer of $\Delta \mathrm{a} / \mathrm{a}=1.8 \cdot 10^{-3}$ (where ' $\mathrm{a}$ ' is the lattice parameter) with the composition $\mathrm{Ga}_{0.51} \mathrm{In}_{0.49} \mathrm{P}$ and a structure factor (S) [23] of $\mathrm{S}=0.4$; indicating a high optical quality (starting) material. PL measurements were performed on the embedded GaInP nanopillar/disk arrays and 'slab' structures for two source wavelengths: 450 (blue) and 532 (green) nm; where Fig. 2(a) shows representative data for the PL emission for a source wavelength of $532 \mathrm{~nm}$. The PL peak shows a full-width-at-half-maximum (FWHM) of $\sim 15 \mathrm{~nm}$ and an emitting wavelength of $\sim 660 \mathrm{~nm}$; where variations in the peak position (657-663 nm) are due to non-uniformity in the original wafer. The nanopillar array shows the highest PL intensity most likely due to a better in-coupling and absorption of the source light and a better out-coupling (light extraction) due to the type of structuring; where the nanopillar arrays can 

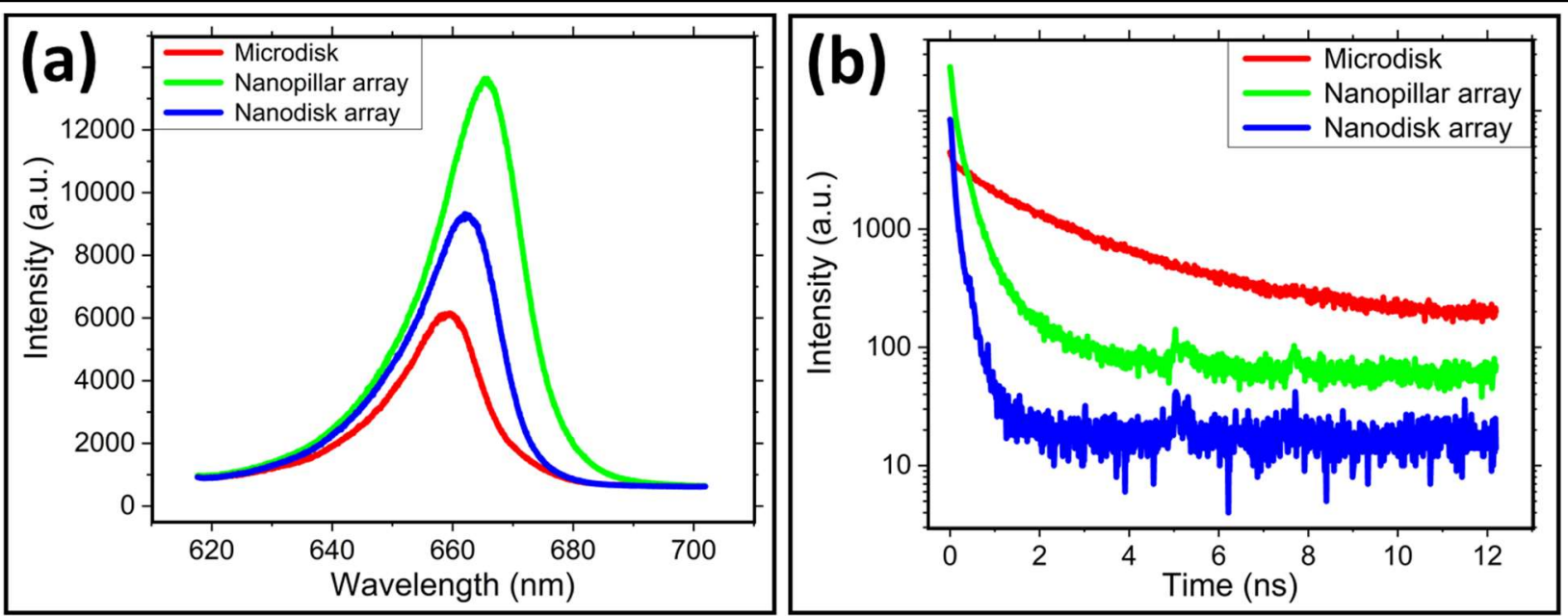

Fig. 2. (a) Photoluminescence (PL) spectra and (b) time-resolved PL (TRPL) spectra for the fabricated (non-passivated) GaInP micro- and nanostructures embedded in a PDMS film for an average power density of $57 \mathrm{~kW} / \mathrm{cm}^{2}$. The variations in the peak positions are due to non-uniformity in the wafer.

absorb $\sim 95 \%$ and $\sim 96 \%$ of the source wavelengths of 450 and $532 \mathrm{~nm}$, respectively, and the (infinite) 'slab' $\sim 83 \%$ and $81 \%$, respectively.

To investigate the influence of the ICP-RIE process on the properties of the GaInP structures, room temperature PL and TRPL measurements were performed in order to investigate the carrier life-time properties and the related PL intensities (before and after processing); where Fig. 2(b) shows the TRPL data related to the PL spectra shown in Fig. 2(a). For these measurements a Ti:Sapphire Chameleon Ultra II laser source was used with femtosecond laser pulses (140 fs FWHM) with a repetition rate of $80 \mathrm{MHz}$ and frequency doubled by a
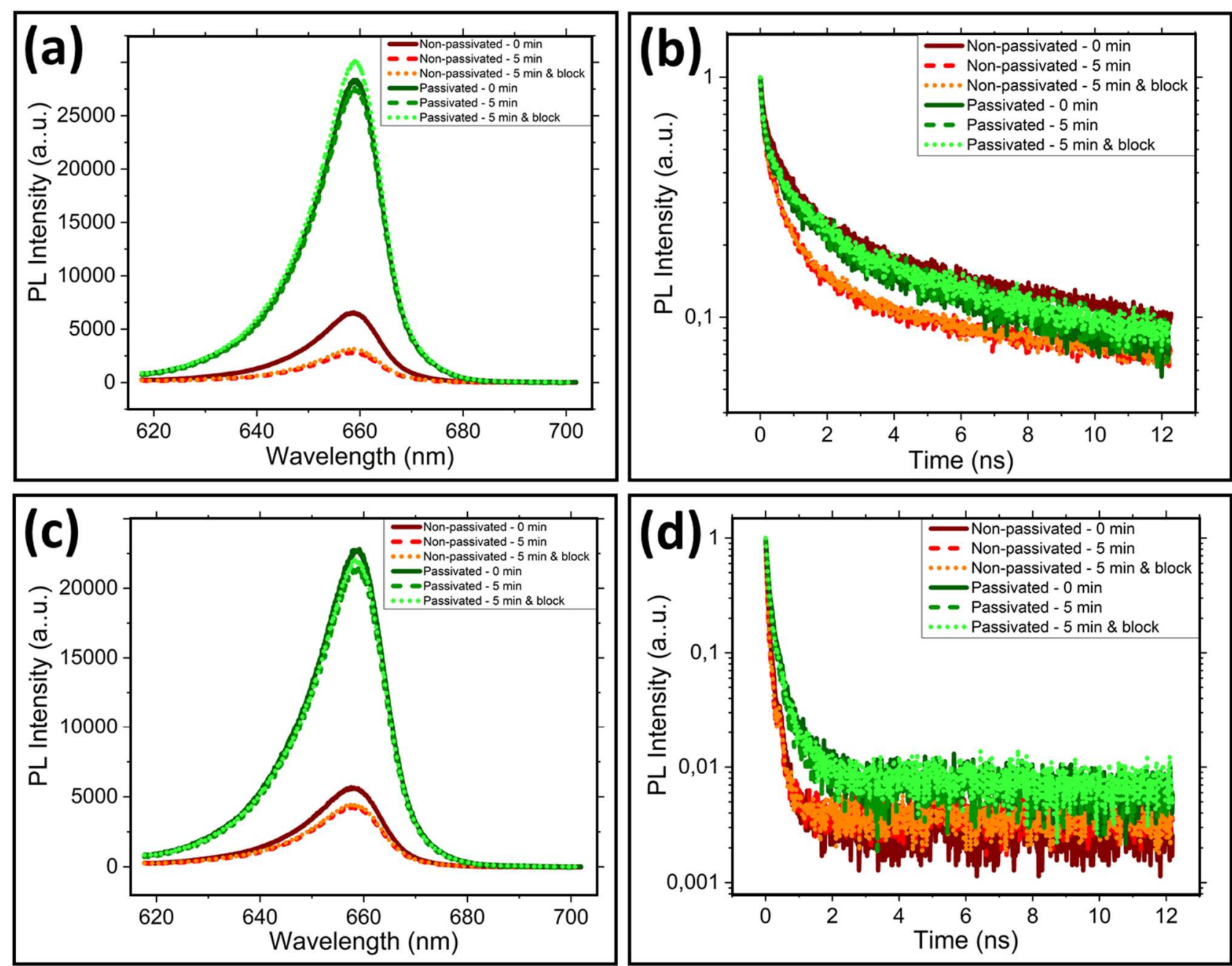

Fig. 3. Photoluminescence (PL) spectra and normalized time-resolved PL (TRPL) spectra for non-passivated and passivated GaInP structures (for an average power density of $57 \mathrm{~kW} / \mathrm{cm}^{2}$ ), related to the occurring PL degradation. The PL and TRPL data for the original GaInP/GaAs substrate, is included in (a) and (b), respectively. The PL and TRPL data for the nanopillar (NP) arrays still on the substrate, is included in (c) and (d), respectively. 


\section{Accepted Manuscript - IEEE copyright}

harmonic generator for two different excitation wavelengths: 450 and $532 \mathrm{~nm}$; for average power densities of 57, 96 and $134 \mathrm{~kW} / \mathrm{cm}^{2}$ and a spot size of $\sim 20 \mu \mathrm{m}$. The TRPL results for the GaInP structures embedded in PDMS, indicate carrier lifetimes of 2-3 ns for the microdisk which indicates a good quality (bulk) material and $\sim 0.5$ and $\sim 0.3 \mathrm{~ns}$ for the nanopillar and nanodisk arrays, respectively; which is quite good for nanopillar/disk structures. A slight improvement ( $30 \%)$ for the carrier lifetimes was observed for lower average excitation powers (from 134 to $57 \mathrm{~kW} / \mathrm{cm}^{2}$ ). For both the non-passivated embedded structures and ICP-RIE etched structures on the substrate, a clear decrease in the PL intensity is observed with time when keeping the laser at the same spot. This phenomenon was observed by first measuring the PL from the start $(0 \mathrm{~min})$ and then keeping the laser at the same spot for 5 minutes $(5 \mathrm{~min})$ followed by measuring the PL again. This degradation is attributed to photo-induced chemical modifications (photodarkening and photobleaching) of the material in ambient air [24] and appears to be irreversible; the PL and TRPL signals do not recover to their original values. The recovery was determined by subsequently blocking the laser for $5 \mathrm{~min}$ ( 5 min \& block) and observe if the PL signal recovers. GaInP structures passivated by a sulphur-oleylamine based solution indicate an improvement of both the PL intensity and carrier lifetimes; where for the nanopillars the PL intensity and carrier lifetimes increased $\sim 5 \mathrm{x}$ and $\sim 20 \%$. In addition, the passivated structures did not show any observable photo-induced PL degradation with time. Representative data for the PL spectra and TRPL data for the original $\mathrm{GaInP} / \mathrm{GaAs}$ substrate and the fabricated $\mathrm{GaInP}$ nanopillar arrays (still on the substrate), are included in Fig. 3; where the 'red' data is related to the non-passivated structures and the 'green' data to the passivated structures. This indicates that surface passivation (electronic and chemical) is beneficial for improving the material quality.

\section{Conclusion}

A top-down method was used based on ICP-RIE to fabricate high optical quality GaInP nanopillar/disk arrays. The structures were embedded in a highly transparent film in order to study their optical characteristics. PL and TRPL measurements were used to determine the PL intensity and carrier lifetimes before (reference 'slab') and after processing (nanopillar/disk structures). A surface passivation treatment was used for improving the PL intensities, increase carrier lifetimes and prevent photo-induced PL degradation by passivating the surface states.

\section{ACKNOWLEDGMENT}

The authors would like to acknowledge the support from the Linné center for advanced optics and photonics (ADOPT; grant number: 349-2007-8664), the Swedish Research Council (VR) and the Swedish Energy Agency (Energimyndigheten; grant number: 45199-1).

\section{REFERENCES}

[1] R. Sridhara, S. M. Frimel, K. P. Roenker, N. Pan, and J. Elliott, "Performance Enhancement of GaInP/GaAs Heterojunction Bipolar Phototransistors Using DC Base Bias," J. Light. Technol., vol. 16(6), pp. 1101-1106, June 1998.

[2] D. Jung, C. A. Parker, J. Ramdani, and S. M. Bedair, "AlGaAs/GaInP heterojunction tunnel diode for cascade solar cell application,” J. Appl. Phys., vol. 74(3), pp. 2090-2093, June 1998.
[3] A. Chen and S. J. Chua, "Two-dimensional AlGaInP/GaInP photonic crystal membrane lasers operating in the visible regime at room temperature," Appl. Phys. Lett., vol. 90, pp. 011113, January 2007.

[4] B. Wang, C. Wang, K. H. Lee, S. Bao, K. E. K. Lee, C. S. Tan, S. F. Yoon, E. A. Fitzgerald, and J. Michel, "Red InGaP light-emitting diodes epitaxially grown on engineered Ge-on-Si substrates," Proc. of SPIE, vol. 9768, pp. 97681J, March 2016.

[5] Y. Wang, R. Zhang, Z. Zhang, B. Qiu, S. Wang, and X. Wu, "Efficiency improvement of GaInP solar cells by broadband omnidirectional antireflection through dielectric composite nanostructures," Sol. Energy Mater. Sol. Cells, vol. 169, pp. 33-39, May 2017.

[6] J. Plá, M. Barrera, and F. Rubinelli, "The influence of the InGaP window layer on the optical and electrical performance of GaAs solar cells," Semicond. Sci. Technol., vol. 22, pp. 1122-1130, September 2007.

[7] J. R. Lothian, J. M. Kuo, F. Ren, and J. Pearton, "Plasma and Wet Chemical Etching of $\operatorname{In}_{0.5} \mathrm{Ga}_{0.5} \mathrm{P}$," Journal of Electronic Materials, vol. 21(4), pp. 441-445, April 1992.

[8] J. W. Wu, C. Y. Chang, E. Y. Chang, S. H. Chang, and K. C. Lin, "Reactive Ion Etching of GaInP, GaAs, and AlGaAs," J. Electrchem. Soc., vol. 142(4), pp. 1340-1343, April 1995.

[9] P. Leerungnawarat, H. Cho, D. C. Hays, J. W. Lee, M. W. Devre, B. H. Reelfs, D. Johnson, J. N. Sasserath, C. R. Abernathy, and S. H. Pearton, "Selectrive Dry Etching of InGaP over GaAs in Inductively Coupled Plasmas," Journal of Electronic Materials, vol. 29(5), pp. 586590, May 2000.

[10] A. Gin, Y. Wei, A. Hood, A. Bajowala, Q. Nguyen, V. Yazdanpanah, and M. Razeghi, "Nanopillars for bandgap-engineering in III-V optoelectronic devices," Proc. of SPIE, vol. 5361, pp. 66-75, June 2004.

[11] Z. Hao, S. Han, F. Ren, B. Xiong, C. Sun, and Y. Luo, "Smooth and Vertical Etching of GaAs/GaInPO/AlGaInP Using Inductively Coupled $\mathrm{CL}_{2} / \mathrm{BCl}_{3} / \mathrm{CH}_{4}$ Plasma," Japanese Journal of Applied Physics, vol. 43(12), pp. 8304-8307, December 2004.

[12] S. Cobrié, S. Bansropun, M. Lecomte, O. Parillaud, S. Cassette, H. Benisty, and J. Nagle, "Optimization of an inductively coupled plasma etching process of $\mathrm{GaInP} / \mathrm{GaAs}$ based material for photonic band gap applications," J. Vac. Sci. Technol. B, vol. 23(4), pp. 1521-1526, July 2005.

[13] N. N. Kornienko, D. D. Whitmore, Y. Yu, S. R. Leone, and P. Yang, "Solution Phase Synthesis of Indium Gallium Phosphide Alloy Nanowires," ACS Nano, vol. 9(4), pp. 3951-3960, April 2015.

[14] D. Jacobsson, J. M. Persson, D. Krieger, T. Etzelstorfer, J. Wallentin, J. B. Wagner, J. Stangl, L. Samuelson, K. Deppert, andM. T. Borgström, "Particle-assisted $\mathrm{Ga}_{\mathrm{x}} \mathrm{In}_{1-\mathrm{x}} \mathrm{P}$ nanowire growth for designed bandgap structures," Nanotechnology, vol. 23, pp. 245601, May 2012.

[15] N. Anttu, V. Dagyte, X. Zeng, G. Otnes, and M. Borgström, "Absorption and transmission of light in III-V nanowire arrays for tandem solar cell applications," Nanotechnology, vol. 28, pp. 205203, April 2017.

[16] A. Berg, Pl Caroff, N. Shahid, M. N. Lockrey, X. Yuan, M. T. Borgström, H. H. Tan, and C. Jagadish, "Growth and optical properties of $\operatorname{In}_{\mathrm{x}} \mathrm{Ga}_{1-\mathrm{x}} \mathrm{P}$ nanowires synthesized by selective-area epitaxy," Nano Research, vol. 10(2), pp. 672-682, February 2017.

[17] E. Barrigón, M. Heurlin, Z. Bi, B. Monemar, and L. Samuelson, "Synthesis and Application of III-V Nanowires," Chem. Rev., vol. 119, pp. 9170-9220, August 2019.

[18] S. Naureen, N. Shahid, R. Sanatinia, and S. Anand, "Top-Down Fabrication of High Quality III-V Nanostructures by Monolayer Controlled Sculpting and Simultaneous Passivation," Adv. Funct. Mater., vol. 23(13), pp. 1620-1627, April 2013.

[19] M. Schubert, V. Gottschalch, C. M. Herzinger, H. Yao, P. G. Snyder, and J. A. Woolam, "Optical constants of $\mathrm{Ga}_{\mathrm{x}} \mathrm{In}_{1-\mathrm{x}} \mathrm{P}$ lattice matched to GaAs,” J. Appl. Phys., vol. 77(7), pp. 3416-3419, January 1995.

[20] P. Thiagarajan, J. F. Schmerge, C. S. Menoni, M. Marconi, O. E. Martinez, J. J. Rocca, M. J. Hafich, H. Y. Lee, and G. Y. Robinson, "Picosecond absorption dynamics of photoexcited InGaP epitaxial films,” Appl. Phys. Lett., vol. 59(1), pp. 90-92, April 1991.

[21] N. M. Haegel, T. J. Mills, M. Talmadge, C. Scandrett, C. L. Frenzen, H. Yoon, C. M. Fetzer, and R. R. King, "Direct imaging of anisotropic minority-carrier diffusion in ordered GaInP," J. Appl. Phys., vol. 105, pp. 023711, January 2009. 


\section{Accepted Manuscript - IEEE copyright}

[22] F. J. Shultes, T. Christian, R. Jones-Albertus, E. Pickett, K. Alberi, B. Fluegel, T. Liu, P. Misra, A. Sukiasyan, H. Yuen, and N. M. Haegel, "Temperature dependence of diffusion length, lifetime and minority electron mobility in GaInP,” Appl. Phys. Lett., vol. 103, pp. 242106, December 2013.

[23] G. R. Moriarty, M. Kildemo, J. T. Beechinor, M. Murtagh, P. V. Kelly, G. M. Crean, and S. W. Bland, "Optical and structural properties of InGaP heterostructures," Thin Solid Films, vol. 364, pp. 244-248, March 2000.

[24] P. Ranga, "Growth and Characterization of III-V Phosphide Nanowires," Master Thesis, Arizona State University, (2016). 